\title{
Rehabilitation der chinoloninduzierten Tendinopathie der Achillessehne ${ }^{1}$
}

Fallstudie

Rehabilitation of Quinolone-Induced Achilles Tendinopathy

\author{
Case Study
}

Autoren

Dario Kohlbrenner ${ }^{1}$, Sandra Schneider ${ }^{2}$, Christian Benden ${ }^{3}$

Institute

1 UniversitätsSpital Zürich, Physiotherapie/Ergotherapie, Zürich, Schweiz

2 Zürcher Fachhochschule für Angewandte Wissenschaften, Institut für Physiotherapie, Winterthur, Schweiz

3 UniversitätsSpital Zürich, Klinik für Pneumologie, Zürich, Schweiz

\section{Schlüsselwörter}

Tendinopathie, Physiotherapie, Rehabilitation, Antibiotika, Fallstudie

Key words

tendinopathy, physiotherapy, rehabilitation, antibiotics, case study

eingereicht 20.09.2017

akzeptiert 21.12.2017

Bibliografie

DOI https://doi.org/10.1055/a-0749-0432

physioscience 2018; 14: 178-185

(c) Georg Thieme Verlag KG, Stuttgart · New York

ISSN 1860-3092

Korrespondenzadresse

Dario Kohlbrenner, MSc PT

UniversitätsSpital Zürich

Klinik für Pneumologie, Rämistr. 100, 8091 Zürich,

Schweiz

Dario.Kohlbrenner@usz.ch

\section{ZUSAMMENFASSUNG}

Hintergrund Der Einsatz von Antibiotika gehört zum medizinischen Alltag. Ein dabei häufig eingesetzter Wirkstoff ist die Gruppe der Chinolone. Physiotherapeutisch relevant sind deren muskuloskeletale Nebenwirkungen, insbesondere auf das Sehnengewebe. Meist sind die Achillessehnen durch Tendinopathien oder Rupturen betroffen. Es besteht ein Mangel

1 Die Fallstudie verfasste D. Kohlbrenner als Leistungsnachweis im Studiengang MSc Physiotherapie der ZHAW Gesundheit, Winterthur. an Literatur bezüglich der Pathophysiologie als auch der Rehabilitation dieser klinischen Muster.

Ziel Diese Fallstudie evaluierte die Anwendung eines exzentrischen Trainingsprotokolls bei chinoloninduzierter Tendinopathie der Achillessehne.

Methode Die physiotherapeutische Rehabilitation einer 48-jährigen Frau mit unilateraler Tendinopathie der Achillessehne unter Einnahme des Antibiotikums Ciprofloxacin nach Lungentransplantation wird beschrieben. Dabei wurde ein kriterienbasierter Ansatz mit ausschließlich Hands-off-Therapie unter Einsatz eines exzentrischen Belastbarkeitsaufbaus der Achillessehne angewendet.

Ergebnisse Nach 9 Einzeltherapien fanden sich Verbesserungen beim 1-Minute-Sitz-Stand-Test, der Range of Motion bei Dorsalextension des oberen Sprunggelenks rechts, beidseitige Kraft von Knieextensoren und -flexoren, Dorsalextensoren und Plantarflexoren des oberen Sprunggelenks, beim VISA-AFragebogen sowie bei Schmerz und Partizipation.

Schlussfolgerungen Bei chinoloninduzierter Tendinopathie der Achillessehne ist ein exzentrisch betontes Trainingsprotokoll durchführbar und wirksam, wobei die zahlreichen Komorbiditäten der primär betroffenen Population zu beachten sind. Da Physiotherapeuten aufgrund der demografischen Entwicklung zunehmend mit hochkomplexen Fällen konfrontiert werden, ist es für sie wichtig, die unerwünschten Wirkungen von Medikamenten zu kennen.

\section{ABSTRACT}

Background The use of antibiotics is a common medical treatment. Frequently used agents are quinolones. Relevant in physiotherapy are their musculoskeletal side effects, predominantly on tendon tissues. The Achilles tendons are usually affected by tendinopathies and ruptures. There is a lack of literature regarding the pathophysiology as well as the rehabilitation of these clinical patterns.

Objective This case study evaluated the use of eccentric training in quinolone-induced Achilles tendinopathy.

Method The physiotherapeutic rehabilitation of a 48-year old woman with unilateral Achilles tendinopathy due to antibiotic ciprofloxacin therapy after lung transplantation is described. A criterion-based therapy regimen with exclusively hands-off 
treatment and focus on eccentric load development of the Achilles tendon was applied.

Results After 9 single-case sessions improvements of the 1-minute-sit-to-stand test, the range of motion in dorsal extension of the right talocrural ankle, the both-sided strength of knee extensors and flexors, dorsal extensors and plantar flexors of the talocrural ankle, the VISA-A questionnaire as well as of pain and participation were found.
Conclusions A heavy load eccentric training protocol is feasible and effective in quinolone-induced Achilles tendinopathy. The several comorbidities of the primarily affected population must, however be considered. Since physiotherapists will increasingly be faced with multi-dimensional cases, it is important for them to know about the unwanted side effects of drugs.

\section{Einleitung}

Der Einsatz von Antibiotika gehört zum medizinischen Alltag. In der Schweiz werden während der stationären Behandlung pro 100 Liegetage 62,9 Tagesdosen und in der ambulanten Behandlung 5,7 Tagesdosen pro 1000 Einwohner und Tag eingenommen [1]. Dabei ist die Gruppe der Chinolone mit $10 \%$ in der stationären Behandlung bzw. 13\% im ambulanten Setting einer der am häufigsten eingesetzten Wirkstoffe [1]. Diese Wirkstoffgruppe kommt primär zur Behandlung von Infektionen des respiratorischen und des Urogenitaltrakts zur Anwendung [2].

Beim Einsatz von Chinolonen sind die muskuloskeletal relevanten Nebenwirkungen, insbesondere auf das Sehnengewebe zu beachten: Die Einnahme verdreifacht das Risiko einer Achillessehnenruptur [3]. Diese gravierende Nebenwirkung kommt bei gesunden Menschen selten vor, das Risiko steigt jedoch bei Patienten mit Nierenfunktionsstörungen, nach Transplantationen, bei einem Alter über 65 Jahre und bei Komedikation mit Glukokortikoiden bis zu einer Odds Ratio von 20 an [2, 4]. Zwar wurden bereits mehrere Fallberichte zur Behandlung nach chinoloninduzierter Achillessehnenruptur publiziert [5], randomisierte Studien liegen jedoch keine vor.

Zu der etwas weniger gravierenden Nebenwirkung, der Tendinopathie der Achillessehne, fehlen verlässliche epidemiologische Untersuchungen. Es wird jedoch eine Häufigkeitsschätzung von 0,14-0,44\% der medikamentös behandelten Patienten angenommen [6]. Bekanntermaßen nimmt die physiotherapeutische Behandlung bei Achillessehnen-Tendinopathien einen wichtigen Stellenwert ein [7]. Da die Pathophysiologie der chinoloninduzierten Achillessehnen-Tendinopathie unklar ist und sich bildgebend noch ungenügend darstellen lässt [6], gestaltet sich die Physiotherapie, insbesondere die initiale Vorgabe der erlaubten Belastung, sehr schwierig. Green [8] wählte in seinem Fallbericht einen zeitbasierten Ansatz mit konsequenter Entlastung durch Gehstützen und einen Fersenkeil.

Im Folgenden wird das kriterienbasierte physiotherapeutische Vorgehen zur Behandlung einer chinoloninduzierten Achillessehnen-Tendinopathie bei einer Patientin nach Lungentransplantation dargestellt.

\section{Methode}

Es wird der Fall einer 48-jährigen Frau beschrieben, die sich 2 Monate nach einer Lungentransplantation und einem nach der
Hospitalisation direkt erfolgten 3-wöchigen stationären Rehabilitationsaufenthalt zur ambulanten Physiotherapie vorstellte.

Die Patientin äußerte neu aufgetretene Schmerzen an der rechten Achillessehne, die sie in ihrem Alltag deutlich einschränkten. Die Schmerzen waren bei aufrechten Aktivitäten stetig vorhanden (numerische Ratingskala [NRS]: 2/10), verschlimmerten sich beim Gehen (NRS: 4/10) und erreichten ihre Spitze beim Treppenheruntersteigen (NRS: 7/10). Die Qualität der Schmerzen beschrieb sie als „reißend, ziehend“. Die Einnahme des Analgetikums Metamizol linderte die Schmerzen, ließ sie aber nicht komplett verschwinden. Wie häufig nach einer Lungentransplantation im Falle einer nachgewiesenen Besiedelung mit dem Bakterium Pseudomonas aeruginosa nahm die Patientin seit der Operation Ciprofloxacin ein [9]. Nach Rücksprache mit dem behandelnden Transplant-Pneumologen bestätigte sich der Verdacht einer chinoloninduzierten Tendinopathie der Achillessehne, und die Antibiotikatherapie mit Ciprofloxacin wurde sofort abgesetzt.

Bei der Inspektion zeigte sich die Achillessehne leicht überwärmt, gerötet, gequollen und druckdolent. Dehnungsreize, auch in passiver Form, provozierten den beschriebenen Schmerz.

Patienten in der ambulanten Rehabilitation nach Lungentransplantation durchlaufen an unserem Zentrum ein standardisiertes physiotherapeutisches Assessment-Verfahren, bei dem Parameter zur isometrischen Kraft der Knieflexoren und -extensoren, Griffkraft, 6-Minuten-Gehtest und 1-Minute-Sitz-Stand-Test erhoben werden. Im beschriebenen Fall wurde das Assessment angepasst und aufgrund der Schmerzen beim Gehen auf den 6-Minuten-Gehtest verzichtet und mit Kraftmessung von Dorsalextension und Plantarflexion des oberen Sprunggelenks beidseits ergänzt (Erfassung der schmerzfrei produzierbaren Kraft).

Die benutzten Verlaufsparameter, geordnet nach den Überkategorien der Internationalen Klassifikation der Funktionsfähigkeit, Behinderung und Gesundheit sind in $>$ Tab. 1 dargestellt. Die Range of Motion wurde passiv bis zur Schmerzprovokation erfasst. Für eine bessere Vergleichbarkeit sind die mit dem Dynamometer MicroFET2 standardisiert gemessenen Kraftwerte prozentual zu den Referenzwerten dargestellt [10].

Das Dynamometer verfügt über eine hohe Inter- und Intrarater-Reliabilität und auch Daten zur kleinsten messbaren Veränderung sind verfügbar [12]. Als Fragebogen kam der VISA-A zum Einsatz, der ebenfalls eine gute Reliabilität aufweist [13] und sich als Verlaufskontrolle bei Achillessehnen-Tendinopathie eignet [14]. Die Resultate der Assessments aus der Befundaufnahme finden sich in $>$ Tab. 2. 
- Tab. 1 Übersicht über alle benutzten Assessments, aufgeteilt nach Kategorien der International Classification of Functioning, Disability and Health (ICF; [11]).

\begin{tabular}{|l|l|l|}
\hline Struktur und Funktion & Aktivität & Partizipation \\
\hline Schmerz (numerische Ratingskala) & 1 Minute Sitz-Stand-Test & Ausflüge mit der Familie im Freien \\
\hline MicroFET2 (Dynamometer) & Treppensteigen & Freundinnen besuchen \\
\hline Range of Motion & VISA-A (Fragebogen) & \\
\hline
\end{tabular}

- Tab. 2 Übersicht der Resultate aller Assessments nach 9 Behandlungen Einzeltherapie und 22 Manuelle-Therapie-Terminen.

\begin{tabular}{|c|c|c|c|}
\hline Assessment & Befundaufnahme & $\begin{array}{l}\text { Abschluss der Behandlung } \\
\text { nach } 12 \text { Wochen }\end{array}$ & Veränderung total \\
\hline $\begin{array}{l}\text { numerische Ratingskala Treppeherunter- } \\
\text { steigen }\end{array}$ & $7 / 10$ & $0 / 10$ & $-7 / 10$ \\
\hline Kraft Knieflexion rechts & $63 \%$ & $75 \%$ & $+12 \%$ \\
\hline Kraft Knieflexion links & $60 \%$ & $76 \%$ & $+16 \%$ \\
\hline Kraft Knieextension rechts & $30 \%$ & $68 \%$ & $+38 \%$ \\
\hline Kraft Knieextension links & $28 \%$ & $70 \%$ & $+42 \%$ \\
\hline $\begin{array}{l}\text { Kraft Dorsalextension oberes } \\
\text { Sprunggelenk rechts }\end{array}$ & $66 \%$ & $85 \%$ & $+19 \%$ \\
\hline $\begin{array}{l}\text { Kraft Dorsalextension oberes } \\
\text { Sprunggelenk links }\end{array}$ & $80 \%$ & $92 \%$ & $+12 \%$ \\
\hline $\begin{array}{l}\text { Kraft Plantarflexion oberes Sprunggelenk } \\
\text { rechts }\end{array}$ & $21 \%$ & $88 \%$ & $+67 \%$ \\
\hline $\begin{array}{l}\text { Kraft Plantarflexion oberes Sprunggelenk } \\
\text { links }\end{array}$ & $79 \%$ & $96 \%$ & $+17 \%$ \\
\hline $\begin{array}{l}\text { Range of Motion Dorsalextension oberes } \\
\text { Sprunggelenk rechts }\end{array}$ & $5^{\circ}$ & $20^{\circ}$ & $+15^{\circ}$ \\
\hline $\begin{array}{l}\text { Range of Motion Dorsalextension oberes } \\
\text { Sprunggelenk links }\end{array}$ & $20^{\circ}$ & $20^{\circ}$ & $0^{\circ}$ \\
\hline $\begin{array}{l}\text { Range of Motion Plantarflexion oberes } \\
\text { Sprunggelenk rechts }\end{array}$ & $35^{\circ}$ & $35^{\circ}$ & $0^{\circ}$ \\
\hline $\begin{array}{l}\text { Range of Motion Plantarflexion oberes } \\
\text { Sprunggelenk links }\end{array}$ & $40^{\circ}$ & $40^{\circ}$ & $0^{\circ}$ \\
\hline 1-Minute-Sitz-Stand-Test & 0 Wiederholungen & 15 Wiederholungen & +15 Wiederholungen \\
\hline VISA-A (Fragebogen) & $2 / 100$ & $100 / 100$ & $+98 / 100$ \\
\hline Ausflüge mit der Familie im Freien & $\begin{array}{l}\text { nur in naher Umgebung } \\
\text { (Spielplatz im Quartier) }\end{array}$ & ohne Einschränkungen & \\
\hline Freundinnen besuchen & $\begin{array}{l}\text { mehr als } 20 \text { Minuten zu Fuß } \\
\text { gehen oder Treppensteigen } \\
\text { sind nicht möglich }\end{array}$ & ohne Einschränkungen & \\
\hline
\end{tabular}

Bezüglich der Kontextfaktoren ergaben sich sich nur wenige relevante Auffälligkeiten. Die Patientin erfuhr eine gute familiäre Einbettung (verheiratet, 2 Kinder im Grundschulalter) und Unterstützung. Im gewohnten häuslichen Umfeld gab es keine Treppen. Aufgrund der Lungentransplantation war sie noch arbeitsunfähig und durfte kein Fahrzeug lenken. Die Arbeitsunfähigkeit dauert nach diesem Eingriff bei gutem Verlauf mindestens 6 Monate an [10].
Ihre beiden Hobbys Radtouren und Wanderungen waren aktuell bei großem Kraftdefizit in den Beinen schon vor den Achillessehnenbeschwerden nicht möglich. Als Ziele nannte sie in der Befundaufnahme nach der ambulanten Rehabilitation, wieder Radtouren und kurze Wanderungen unternehmen zu wollen (ca. 2 Stunden ohne starke Steigungen) und nach 6 Monaten wieder in ihrem bisherigen Beruf als Architektin arbeiten zu können. An der aktuellen Situation mit den Achillessehnenschmerzen störte 
Polymedikation

(Kortison, Immunsuppressiva,

antibiotische Prophylaxe)

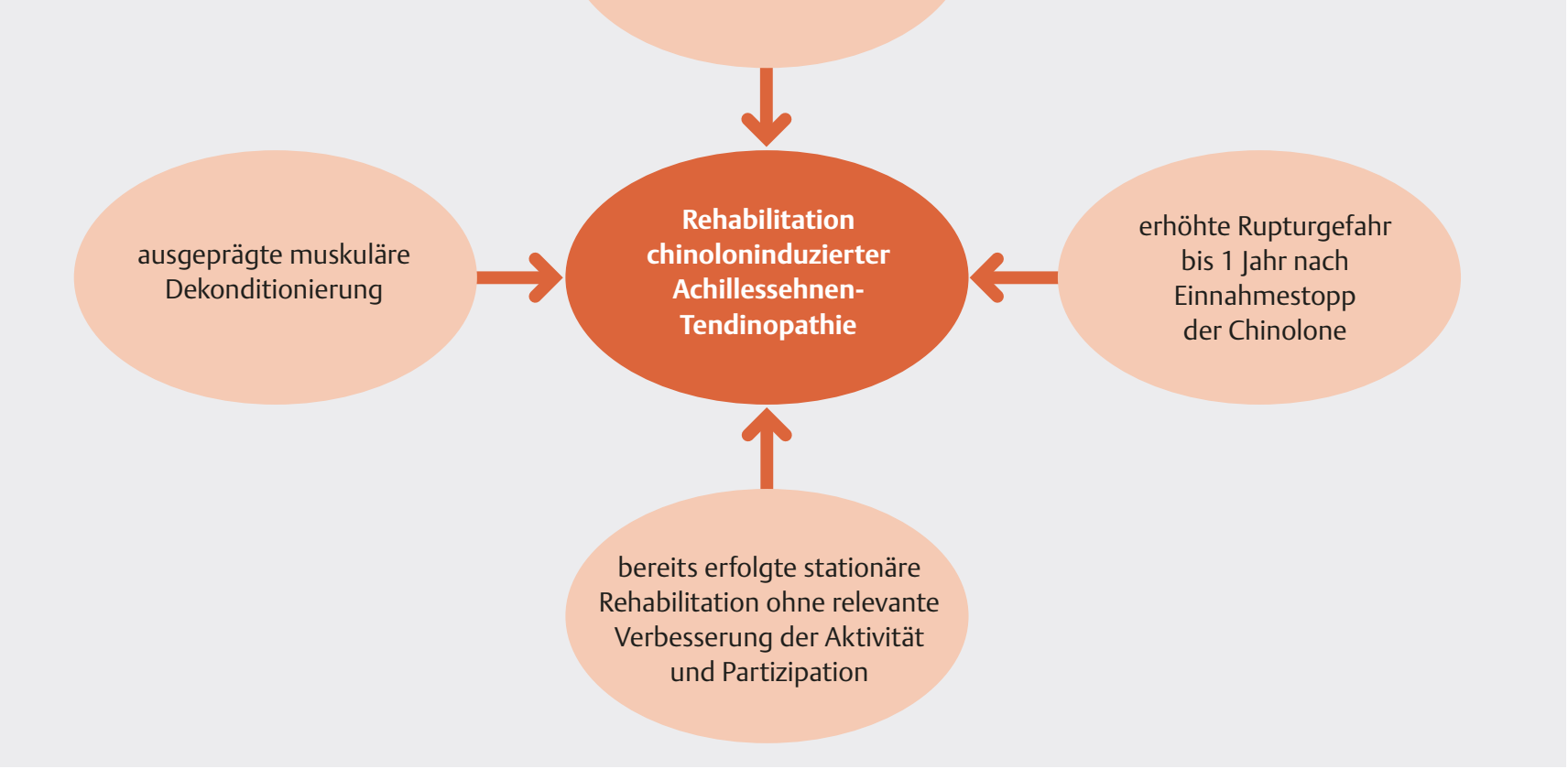

- Abb. 1 Die wichtigsten beitragenden Faktoren zur Erstellung des Rehabilitationsplans. (Quelle: D. Kohlbrenner; graf. Umsetzung: Thieme Gruppe)

sie vor allem, dass das Spielen im Freien mit den Kindern und das Besuchen ihrer Freundinnen (zu Fuß und an einigen Orten sind Treppen zu steigen) stark eingeschränkt waren.

Zusammenfassend präsentierte sich eine muskulär stark eingeschränkte Patientin mit einer chinoloninduzierten Tendinopathie der rechten Achillessehne, die 8 Wochen nach Einnahmestart von Ciprofloxacin auftrat. Die Funktion des Lungentransplantats war optimal, ein respiratorisches oder kardiales Begleitproblem bestand nicht. Es galt jedoch zu bedenken, dass Patienten nach Lungentransplantation in der Regel längerfristig 3 verschiedene immunsupprimierende Medikamente einnehmen [9]. Diese bedingen in der Therapie ein außerordentlich hygienisches Vorgehen; ursächlich schwächen sie aber das Bindegewebe, und insbesondere das als Immunsuppressivum verwendete Kortison hemmt zusätzlich den Kraftaufbau. Bei den betroffenen Patienten ist mit verlangsamter Wundheilung und somit längerem Rehabilitationsverlauf zu rechnen als bei nicht immunsupprimierten Patienten.

\section{Interventionen}

In Anbetracht der ausgeprägten funktionellen Einschränkungen der Patientin erfolgte die Entscheidung für ein progressiveres Vorgehen wie das von Greene [7] beschriebene. Dabei geht der vorliegende Bericht hauptsächlich auf die Interventionen zur Behandlung der Tendinopathie ein.
Aufgrund der unklaren Pathophysiologie wurden ein kriterienbasierter Behandlungsansatz gewählt und die aktuelle Evidenz zur Sehnenrehabilitation einbezogen. Die wichtigsten beitragenden Faktoren, die die Erstellung des Rehabilitationsplanes beeinflussten, zeigt $>$ Abb. 1.

In der 1. Phase stand die Entzündung im Vordergrund, die durch Überlastung der bereits degenerativ veränderten und folglich belastbarkeitsreduzierten Sehne auftrat. Die Patientin wurde im Detail mehrfach informiert, was ihr Problem auslöste und in welchem Rahmen sie aktuell ihre Achillessehne belasten durfte. Zur Schmerzlinderung konnte sie nach Bedarf kurze Eisanwendungen im schmerzenden und geröteten Gebiet vornehmen. Weiter wurde sie angewiesen, die Sehne initial zu schonen, nur die nötigsten Strecken zu Fuß zurückzulegen und möglichst Treppen zu vermeiden. Zur Reduktion der longitudinalen Belastung der Sehne wurde zur Limitierung der Dorsalextension ein starres Tape angelegt. Unter diesem Management besserten sich die Entzündungssymptomatik und die Schmerzintensität innerhalb von 6 Tagen so weit, dass die aktive Rehabilitation beginnen konnte.

In der 2. Phase bestand das primäre Ziel im Erreichen der vollen schmerzfreien Range of Motion. Als Intervention wurde ein Heimprogramm erstellt, das initial aus unbelasteten aktiven Bewegungsübungen des betroffenen Fußes bestand und später durch Einbauen von belasteten Dehnungsübungen intensiviert wurde. Alle Übungen durften bis zur Schmerzgrenze durchgeführt werden. 
- Tab. 3 Exzentrisches Trainingsprotokoll über 12 Wochen.

\begin{tabular}{|c|c|}
\hline Belastungsvariable & exzentrische Calf-Raises \\
\hline Übungsausführung & $\begin{array}{l}\text { Calf-Raises mit Festhalten auf einer Stufe. } \\
\text { Die konzentrische Phase wird zweibeinig, die exzentrische nur mit dem betroffenen Bein ausgeführt. } \\
\text { Das Knie gestreckt bzw. leicht flektiert halten. }\end{array}$ \\
\hline Häufigkeit & 2-mal täglich (1-mal gestrecktes und 1-mal leicht flektiertes Knie) \\
\hline Wiederholungen & 3 Sätze mit 15 Wiederholungen \\
\hline Rhythmus & $\begin{array}{l}\text { Konzentrik: } 1 \text { Sekunde } \\
\text { Pause: } 1 \text { Sekunde } \\
\text { Exzentrik: } 6 \text { Sekunden }\end{array}$ \\
\hline Zusatzgewicht & $\begin{array}{l}\text { über Rucksack Gewicht hinzufügen, sodass } 15 \text { Wiederholungen ohne Schmerz (numerische Ratingskala: > 5/10) } \\
\text { möglich sind }\end{array}$ \\
\hline $\begin{array}{l}\text { Abbruchkriterien (erforder- } \\
\text { liche Maßnahmen beim } \\
\text { Auftreten) }\end{array}$ & $\begin{array}{l}\text { Entzündungszeichen (Information an Physiotherapeuten) } \\
\text { Schmerzen beim Training: numerische Ratingskala > 5/10 (Reduktion Zusatzgewicht) } \\
\text { erhöhte Schmerzen > } 1 \text { Stunde nach dem Training (Reduktion Zusatzgewicht) }\end{array}$ \\
\hline
\end{tabular}

Das Erreichen der vollen schmerzfreien Beweglichkeit dauerte 2 Wochen. Bis zu diesem Zeitpunkt nahm die Patientin einmal pro Woche an einer Einzeltherapie teil. Als zusätzliches aerobes Training übte sie täglich 2-mal 15 Minuten auf dem Fahrradergometer, wobei sie für ein schmerzfreies Training den rechten Fuß mit dem Mittelfuß/Kalkaneus auf die Pedale platzierte.

Neben der sehnenbezogenen Rehabilitation fand ein Krafttrainingsaufbau für Rumpf, obere Extremität und - soweit möglich auch untere Extremität bis zur intensiven Rekrutierungsmethode (KRS 4) statt [15]. Die vorherigen Stufen konnten sie nach Auskunft der stationären Rehabilitation schon erarbeiten. Allerdings wies die Kraft der unteren Extremitäten beim Start der ambulanten Rehabilitation im funktionellen Krafttest (1-Minute-SitzStand-Test) und auch in der Dynamometrie noch immer eine ausgeprägte Schwäche auf.

Die 3. und abschließende Phase bestand im Belastbarkeitsaufbau der betroffenen Sehne. Da auch auf der linken Seite noch eine subjektiv relevante Schwäche vorhanden war, führte die Patientin hierbei bilateral ein intensives exzentrisches Trainingsprotokoll durch ( Tab. 3; [7]). Das Trainingsprogramm wurde in Anbetracht der ausgeprägten muskulären Schwäche der gesamten unteren Extremitätenmuskulatur zugunsten eines exzentrisch betonten Trainingsansatzes verändert, da dieser Ansatz bei respiratorisch erkrankten Patienten einen höheren Effekt auf die Steigerung der Maximalkraft als konventionelles Krafttraining zeigt [16, 17]. Zusätzlich lag ein weiterer Schwerpunkt auf dem Rumpfkrafttraining, weil limitierte Rumpfkraft die Leistung in funktionellen Leistungstests beeinflusst [18] und somit als klinisch relevant gilt.

Zum anfänglichen Erlernen der korrekten Trainingstechnik, zum detaillierten Ausarbeiten des Trainingsplanes und zur Kontrolle der Reaktion des Gewebes auf die gesteigerte, intensive Belastung wurden zu Beginn 2 Einzeltherapien pro Woche geplant. Die Patientin erhielt zudem genaue Informationen über das Protokoll und die erwünschten und unerwünschten Reaktionen. Da noch bis zu 1 Jahr nach Beginn der Chinolontherapie trotz sofortigem Absetzen des Medikamentes ein erhöhtes Rupturrisiko der Achillessehne besteht $[19,20]$, sollte die Patientin bei den geringsten Anzeichen einer Verschlechterung das Programm abbrechen und ihren Physiotherapeuten informieren.

Nach 3 Wochen konnte die Therapiefrequenz auf eine Behandlung pro Woche reduziert werden, wobei der Fokus auf den restlichen Problembereichen und nicht mehr auf der Achillessehne lag. Richtlinienkonform erfolgte die konsequente Weiterführung des exzentrischen Trainingsprotokolls zur Belastbarkeitssteigerung der Achillessehne für 12 Wochen [7]. Bei der jungen Patientin mit hoher Motivation konnte das Programm ab der 5 . Woche in einem Setting der medizinischen Trainingstherapie stattfinden, um auch den weiter bestehenden muskulären Defiziten gerecht zu werden. Insgesamt waren 9 Sitzungen Einzeltherapie nötig, bis die Therapie abgeschlossen werden konnte und die Patientin unter physiotherapeutischer Supervision den Belastbarkeitsaufbau in einer medizinischen Trainingstherapie selbstständig weiter ausführte.

\section{Ergebnisse}

Eine Übersicht aller Assessments zu den 2 Messzeitpunkten ist in - Tab. 2 dargestellt. Zusätzlich illustriert die Veränderungen der isometrischen Maximalkraft.

Bei allen gemessenen Parametern ist eine Verbesserung zu beobachten, wobei die Verlaufszeichen aus der Kategorie der Partizipation nicht weiter quantifiziert wurden. Bei der Beweglichkeit des oberen Sprunggelenks von Dorsalextension und Plantarflexion links sowie Plantarflexion rechts ergab sich keine Veränderung. Hier war bereits beim Befund volle physiologische Beweglichkeit vorhanden.

Der deutlichste Kraftfortschritt zeigte sich bei den Plantarflexoren rechts und den Knieextensoren beiderseits. Diese Werte wiesen beim Befund auf deutliche Defizite hin, und die Veränderung für die Plantarflexion ging mit der deutlichen Schmerzabnahme einher.

Aufgrund regelmäßig stattfindender ärztlicher Sprechstunden nach Lungentransplantation bestand die Möglichkeit, den Verlauf 


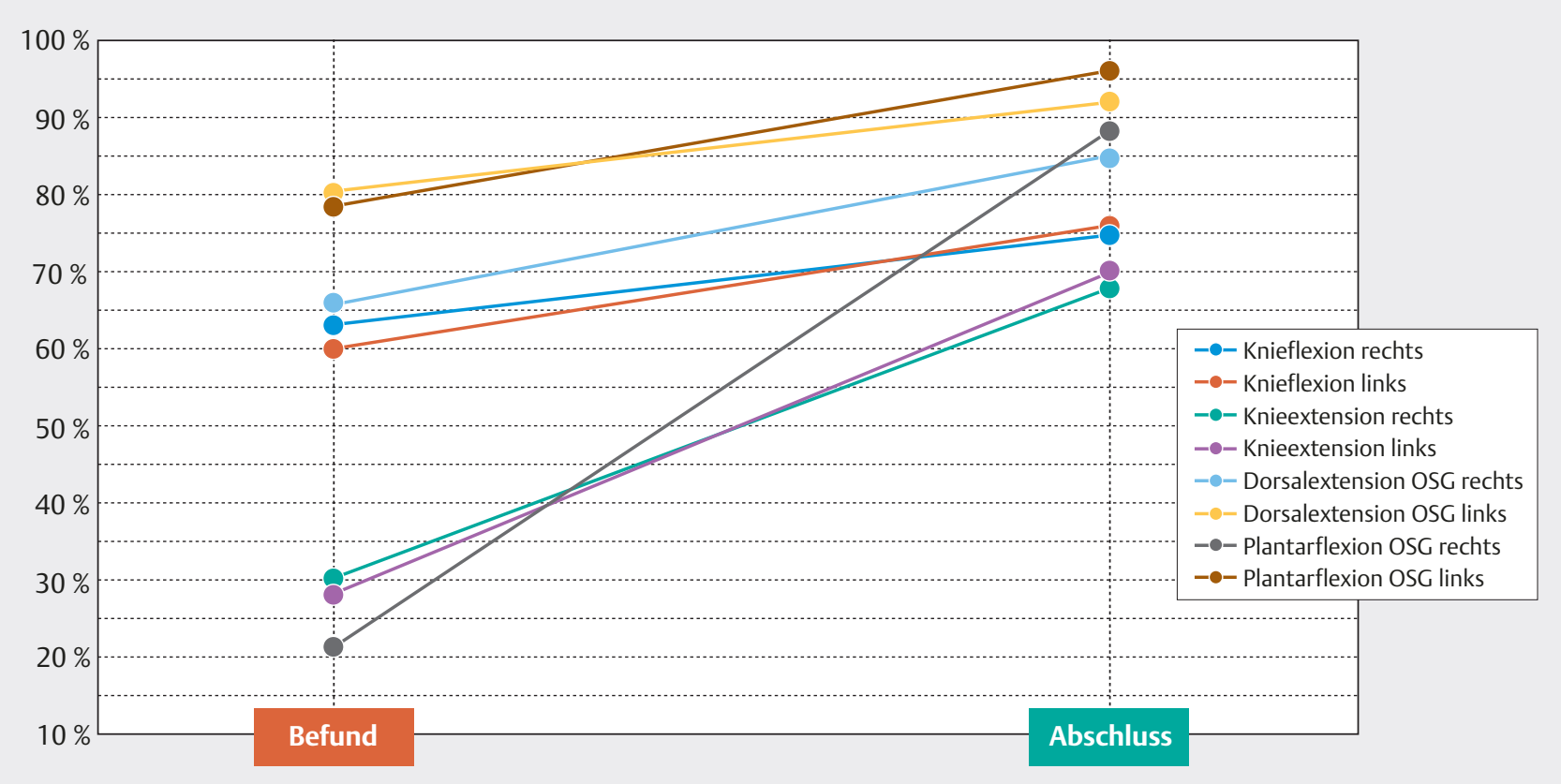

- Abb. 2 Veränderung der isometrischen Kraftwerte. (Quelle: D. Kohlbrenner; graf. Umsetzung: Thieme Gruppe)

der Patientin bis 2 Jahre nach dem Ereignis weiterzuverfolgen. Sie erlitt in dieser Zeit keine neuen Episoden einer Tendinopathie oder Ruptur der Achillessehne.

\section{Diskussion}

Dieser Fallbericht beschreibt einen positiven Rehabilitationsverlauf nach einer chinoloninduzierten Achillessehnen-Tendinopathie. Das Design einer Fallstudie eignet sich insbesondere zur Darstellung des Managements einer seltenen Pathologie, wenn wenig Literatur vorhanden ist. Es ist jedoch zu beachten, dass die Resultate kaum generalisierbar und weitere Studien mit Kontrollpatienten notwendig sind.

Die mangelnde Studienlage bezüglich der Pathophysiologie des Krankheitsbildes stellt das Behandlungsteam vor besondere Herausforderungen und bedingt intensive Absprachen sowie den Einsatz valider und reliabler Verlaufsparameter. Obwohl in diesem Fall erfolgreich ein richtlinienkonformes Protokoll zur Rehabilitation von Achillessehnen-Tendinopathien ohne medikamentösen Auslöser eingesetzt wurde, lässt sich nicht schlussfolgern, dass sich die klinischen Muster ähnlich oder gar gleich sind. Aufgrund der in der Einleitung beschriebenen Risikofaktoren kann hergeleitet werden, dass die betroffene Patientengruppe tendenziell unter zahlreichen Komorbiditäten leidet und langfristig viele Medikamente einnimmt. Daher ist der vorliegende Bericht ein relevantes Beispiel für dieses Krankheitsbild, da zusätzlich zum schmerzhaften Problem noch die ausgeprägte Muskelschwäche zu berücksichtigen war.

Durch die steigende Lebenserwartung und die Fortschritte der hochspezialisierten Medizin ist zu erwarten, dass auf Physiotherapeuten in der Zukunft zunehmend ähnliche Fälle hochkomplexer muskuloskeletaler Rehabilitation zukommen.
Es ist infrage zu stellen, ob die hier beschriebene vulnerable Population ein derart aufwendiges und hartes Trainingsprogramm wie das benutzte exzentrische Protokoll zur Steigerung der Belastbarkeit der Achillessehne neben weiteren notwendigen Übungen/Therapien oder gar während einer akuten Verschlechterung des Gesundheitszustands bzw. eines Spitalaufenthalts durchführen kann.

Der vorliegende Bericht beschreibt dagegen einen komplikationslosen Verlauf. Als wichtiger beitragender Faktor war bei der Patientin zudem eine hohe Selbstwirksamkeit und Motivation vorhanden.

Wie von Greene [8] beschrieben, wurde auch hier initial auf Protektion der Sehne geachtet, um eine Ruptur zu verhindern und die Wundheilung nicht zu verzögern. Im vorliegenden Fall war diese Phase jedoch deutlich kürzer und weniger protektiv gestaltet. Im Rahmen der allgemeinen Dekonditionierung und der höchstwahrscheinlich schon vorhandenen generalisierten Gewebeatrophie durch die lange präoperative Aktivitätseinschränkung ist die kriterienbasierte Rehabilitation zu bevorzugen. Eine erneute längere Immobilisation hätte die Partizipation der Patientin noch einmal deutlich reduziert, die gesamte Rehabilitation verlängert und auch zu einer erhöhten Gefahr für respiratorische Komplikationen geführt.

Mit Rücksicht auf den individuellen Allgemeinzustand der Patienten ist folglich ein progressives, kriterienbasiertes Rehabilitationsprotokoll bei chinoloninduzierter Achillessehnen-Tendinopathie sicher und praktikabel.

Im Vergleich zu Greene [8] fällt zudem auf, dass die Patientin im vorliegenden Fall nach Start der Medikation eine deutlich längere beschwerdefreie Phase hatte. Sie entwickelte erst nach 2 Monaten Therapiedauer Beschwerden, während der von Greene [8] beschriebene Patient bereits nach 3 Tagen akute Symptome 
aufwies. Bei der Untersuchung dieses Aspekts durch Chhajed et al. [20] für eine Population lungentransplantatierter Patienten wies das Auftreten der Symptome keine Korrelation zur verabreichten Dosis und zur Einnahmedauer auf [20]. So können auch noch längere Zeit nach Sistieren der Einnahme chinoloninduzierte Achillessehnen-Tendinopathien und -rupturen entstehen.

Aus bislang unbekannten Gründen besteht bis 1 Jahr nach Absetzen der Medikation und unabhängig von der verschriebenen Dosis und Dauer der Einnahme ein erhöhtes Rupturrisiko [20]. Dies blieb im hier beschriebenen Fall glücklicherweise aus. Möglicherweise verbesserte das benutzte Protokoll die Belastbarkeit der Achillessehne so weit, dass die toxischen Effekte der Chinolone auf das Gewebe [6] kompensiert wurden oder auch das Fortschreiten der Gewebeatrophie aufhalten konnten. Diese Hypothesen lassen sich jedoch mit dem verwendeten Design nicht beantworten.

Anhand der vorhandenen Literatur bei nicht medikamentös induzierten Achillessehnen-Tendinopathien wurde ein Hands-offBehandlungsansatz gewählt [21]. Dies hatte den Vorteil, dass zusätzlich Zeit für die Behandlung bzw. das Training der weiteren Defizite blieb. Außerdem konnte damit bereits nach 9 Behandlungen zu einer medizinischen Trainingstherapie übergegangen werden. Das benutzte Protokoll zur Achillessehnen-Rehabilitation ließ sich optimal mit dem exzentrischen Trainingsansatz der unteren Extremität kombinieren, welches bei stark dekonditionierten Patienten starke Effekte zeigt [17].

Um die Pathophysiologie der chinoloninduzierten Achillessehnen-Tendinopathie zu spezifizieren und anschließend idealerweise in randomisierten Interventionsstudien entsprechende Behandlungsmöglichkeiten prüfen zu können, sind weitere Studien notwendig. Zusätzlich besteht Bedarf an der Publikation von Fallberichten über das Management bei komplikationsreicherem Verlauf.

\section{Schlussfolgerungen}

Diese Fallstudie beschreibt eine seltene Diagnose, deren Kenntnis jedoch für Physiotherapeuten wichtig ist, da sie häufig als erste von Patienten über neu aufgetretene Schmerzen informiert werden.

Die Rehabilitation der chinoloninduzierten AchillessehnenTendinopathie kann nur erfolgreich sein, wenn die Einnahme der Medikation sistiert wird. Dies verdeutlicht die Wichtigkeit, dass Physiotherapeuten in der Anamnese Medikamente erfragen und deren Wirkungen sowie unerwünschten Nebenwirkungen kennen und differenzieren können. So kann eine effektive und patientenorientierte Rücksprache mit dem behandelnden Arzt erfolgen.

Der Bericht legt die Argumentation und das Clinical Reasoning dar, welches zur Anwendung einer evidenzbasierten Therapiemethode bei einem ähnlichen klinischen Muster führt, wenn noch keine evidenzbasierten Behandlungsmöglichkeiten vorliegen. Dies kann eine Basis zu weiteren kontrollierten und randomisierten Studien legen und schlussendlich das klinische Vorgehen nachhaltig beeinflussen.
Die Wahl eines progressiveren und kriterienbasierten Vorgehens ist zentraler Inhalt der Fallbeschreibung. Die Wahl dient mit einer rascheren Rückkehr in die angestrebte Aktivität und Funktion sowohl der Patientin als auch der Allgemeinheit durch deutlich weniger Behandlungen und damit geringere Kosten.

Interessenkonflikt

Die Autoren geben an, dass kein Interessenkonflikt besteht.

Literatur

[1] Bundesamt für Gesundheit (BAG), Bundesamt für Lebensmittelsicherheit und Veterinärwesen (Hrsg). Swiss Antibiotic Resistance Report 2016. Bern: BAG. 2016

[2] Stahlmann R, Lode HM. Risks associated with the therapeutic use of fluoroquinolones. Expert Opinion on Drug Safety 2013; 12: 497-505

[3] Sode J, Obel N, Hallas J et al. Use of fluroquinolone and risk of Achilles tendon rupture: a population-based cohort study. Eur J Clin Pharmacol 2007; 63: 499-503

[4] Van der Linden PD, Sturkenboom MCJM, Herings RMC et al. Increased risk of Achilles tendon rupture with quinolone antibacterial use, especially in elderly patients taking oral corticosteroids. Arch Intern Med 2003; 163: $1801-1807$

[5] Damuth E, Heidelbaugh J, Malani PN et al. An elderly patient with fluoroquinolone-associated achilles tendinitis. Am J Geriatr Pharmacother 2008; 6: $264-268$

[6] Lang TR, Cook J, Rio E et al. What tendon pathology is seen on imaging in people who have taken fluoroquinolones? A systematic review. Fundam Clin Pharmacol 2017; 31: 4-16

[7] Carcia CR, Martin RL, Houck J et al. Achilles pain, stiffness, and muscle power deficits: achilles tendinitis. J Orthop Sports Phys Ther 2010; 40: A1-A26

[8] Greene BL. Physical therapist management of fluoroquinolone-induced Achilles tendinopathy. Phys Ther 2002; 82: 1224-1231

[9] Schuurmans MM, Benden C, Inci I. Practical approach to early postoperative management of lung transplant recipients. Swiss Med Wkly 2013; 143: w13773

[10] Douma RK, Soer R, Krijnen WP et al. Reference values for isometric muscle force among workers for the Netherlands: a comparison of reference values. BMC Sports Science, Medicine and Rehabilitation 2014; 6: 10

[11] World Health Organization (WHO). International Classification of Functioning, Disability and Health (ICF). www.who.int/classifications/icf/ en (11.09.2018)

[12] Buckinx F, Croisier JL, Reginster JY et al. Reliability of muscle strength measures obtained with a hand-held dynamometer in an elderly population. Clin Physiol Funct Imaging 2015; 30: 3

[13] Robinson JM, Cook JL, Purdam C et al. The VISA-A questionnaire: a valid and reliable index of the clinical severity of Achilles tendinopathy. $\mathrm{Br}$ J Sports Med 2001; 35: 335-341

[14] Silbernagel KG, Thomee R, Eriksson BI et al. Continued sports activity, using a pain-monitoring model, during rehabilitation in patients with Achilles tendinopathy: a randomized controlled study. Am J Sports Med 2007; 35: 897-906

[15] Bant H. Sportphysiotherapie. Stuttgart: Thieme. 2011

[16] Butcher S], Pikaluk B], Chura RL et al. Associations between isokinetic muscle strength, high-level functional performance, and physiological parameters in patients with chronic obstructive pulmonary disease. Int J Chron Obstruct Pulmon Dis 2012; 7: 537-542 
[17] Roig M, O’Brien K, Kirk G et al. The effects of eccentric versus concentric resistance training on muscle strength and mass in healthy adults: a systematic review with meta-analysis. Br J Sports Med 2009; 43: 556 568

[18] Janssens L, Brumagne S, McConnell AK et al. Impaired postural control reduces sit-to-stand-to-sit performance in individuals with chronic obstructive pulmonary disease. PLoS One 2014; 9: e88247
[19] Khaliq Y, Zhanel GG. Fluoroquinolone-associated tendinopathy: a critical review of the literature. Clin Infect Dis 2003; 36: 1404-1410

[20] Chhajed PN, Plit ML, Hopkins PM et al. Achilles tendon disease in lung transplant recipients: association with ciprofloxacin. Eur Resp J 2002; 19: 469-471

[21] Silbernagel KG, Brorsson A, Lundberg M. The majority of patients with Achilles tendinopathy recover fully when treated with exercise alone: a 5-year follow-up. Am J Sports Med 2011; 39: 607-613 